\title{
Evaluación positiva de medicamentos: septiembre/octubre/noviembre 2014
}

\author{
Tabakov A. ${ }^{1}$, Aparicio Hernández R. ${ }^{1}$, Pérez Morán MJ. ${ }^{2}$, Prats Olivan P. ${ }^{3}$ \\ Sanid. mil. 2015; 71 (1): 35-43; ISSN: 1887-8571
}

\section{RESUMEN}

Se reseñan los medicamentos evaluados y con dictamen positivo por comisión de expertos de la Agencia Española de Medicamentos y Productos Sanitarios o de la Agencia Europea del Medicamento hecho públicos en septiembre, octubre y noviembre de 2014, y considerados de mayor interés para el profesional sanitario. Se trata de opiniones técnicas positivas que son previas a la autorización y puesta en el mercado del medicamento.

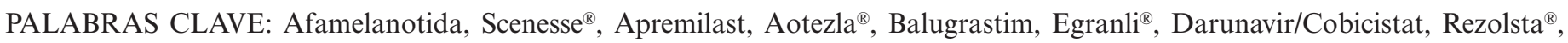
Dasabuvir Sódico, Exviera ${ }^{\circledR}$, Dulaglutida Trulicity ${ }^{\circledR}$, Eliglustat, Cerdelga ${ }^{\circledR}$, Estrógenos Conjugados/Bazedoxifeno, Duavive ${ }^{\circledR}$, Ketoconazol, Ketoconazol Hra Pharma ${ }^{\circledR}$, Naloxegol, Moventig ${ }^{\circledR}$, Nintedanib, Vargatef ${ }^{\circledR}$, Ofev $^{\circledR}$, Nonacog Gamma, Rixubis ${ }^{\circledR}$, Olaparib, Lynparza $^{\circledR}$, Ombitasvir/Paritaprevir/Ritonavir,Viekirax ${ }^{\circledR}$, Ospemifeno, Senshio ${ }^{\circledR}$, Ramucirumab, Cyramza ${ }^{\circledR}$, Secukinumab, Consen$\operatorname{tyx}^{\circledR}$, Sofosbuvir/Ledipasvir, Harvoni ${ }^{\circledR}$, Tilmanocept, Lymphoseek ${ }^{\circledR}$, Vorapaxar,Zontivity ${ }^{\circledR}$.

\section{Possitive assesment of drugs: September, October and November 2014}

SUMMARY: The drugs assessed by the Spanish Agency for Medicines and Health Products or European Medicines Agency made public in September, October and November of 2014, and considered of interest to the healthcare profesional, are reviewed. These are positive technical reports prior to the authorization and placing on the market of the product.

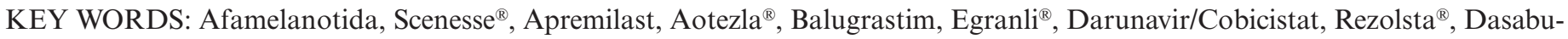
vir Sódico, Exviera ${ }^{\circledR}$, Dulaglutida Trulicity ${ }^{\circledR}$, Eliglustat, Cerdelga ${ }^{\circledR}$, Estrógenos Conjugados/Bazedoxifeno, Duavive ${ }^{\circledR}$, Ketoconazol, Ketoconazol Hra Pharma ${ }^{\circledR}$, Naloxegol, Moventig ${ }^{\circledR}$, Nintedanib, Vargatef ${ }^{\circledR}$, Ofev $^{\circledR}$, Nonacog Gamma, Rixubis ${ }^{\circledR}$, Olaparib, Lynparza $^{\circledR}$, Ombitasvir/Paritaprevir/Ritonavir,Viekirax ${ }^{\circledR}$, Ospemifeno, Senshio ${ }^{\circledR}$, Ramucirumab, Cyramza ${ }^{\circledR}$, Secukinumab, Consentyx ${ }^{\circledR}$, Sofosbuvir/Ledipasvir, Harvoni ${ }^{\circledR}$, Tilmanocept, Lymphoseek ${ }^{\circledR}$, Vorapaxar,Zontivity ${ }^{\circledR}$.

\section{AFAMELANOTIDA (SCENESSE $\left.)^{\circledR}\right)^{1-4}$}

La protoporfiria eritropoyética (PPE) es una porfiria hereditaria poco frecuente que se caracteriza por incremento de protoporfirina que se acumula en los hematíes, en el hígado y en la piel. Por este motivo, la exposición al sol produce una sensación de hormigueo, picor o quemazón en la piel, que puede estar asociada a enrojecimiento e hinchazón. El tratamiento actual se basa en los productos que contienen beta-caroteno, en la fototerapia y en los antihistamínicos.

Afamelanotida es un análogo sintético de la hormona estimulante de melanocitos alfa que actúa como un agonista del receptor de melanocortina-1 estimulando así la melanogénesis. De esta manera, afamelanotida disminuye la sensibilidad a la luz, lo que se

\footnotetext{
${ }^{1}$ Médico Interno Residente de Farmacología Clínica. Servicio de Farmacología Clínica. ${ }^{2}$ Cap. Farmacéutico. Servicio de Farmacia Hospitalaria.

${ }^{3}$ Cte. Farmacéutico. Servicio de Farmacia Hospitalaria.

Hospital Central de la Defensa Gómez Ulla. Madrid. España.
}

Dirección para correspondencia: Aleksandra Tabakov. Servicio de Farmacología Clínica. Hospital Central de la Defensa Gómez Ulla. Glorieta del Ejército 1. 28047 Madrid. Email: atab000@oc.mde.es

Recibido: 14 de enero de 2015

Aceptado: 23 de enero de 2015 traduce en un aumento limitado de tiempo que los pacientes con PPE pueden exponerse a la luz del día o a la luz solar.

La indicación aprobada es prevención de la fototoxicidad en pacientes adultos con protoporfiria eritropoyética (PPE). Afamelanotida está disponible como implante subcutáneo de $16 \mathrm{mg}$.

Los datos de eficacia y seguridad provienen principalmente de un estudio fase III, aleatorizado, multicéntrico, doble ciego, controlado con placebo, de seis meses de duración, que ha incluido 87 pacientes adultos con PPE. Los pacientes recibieron implantes de afamelanotida $(n=45)$ o de placebo $(n=42)$ cada dos meses (días 0,60 y 120). La visita de seguridad estaba programada para el día 210. El objetivo primario fue establecer la medida en que los pacientes se expusieron a la luz sol directa entre las 10:00 y 18:00 sin presentar dolor, observándose una fuerte tendencia hacia una mayor exposición a la luz directa en el grupo activo $(64,13$ horas frente a 47,5 horas, $p=0.107$ ). La tolerancia a luz directa tras foto-provocación en el laboratorio también ha sido significativamente mayor en el grupo con tratamiento activo. Las reacciones adversas más comunes observadas durante el desarrollo clínico fueron: náuseas, nasofaringitis, cefalea, dolor abdominal, fatiga y somnolencia.

Scenesse ${ }^{\circledR}$ fue designado como medicamento huérfano el 8 de mayo de 2008; el 23 de octubre de 2014, el CHMP ha recomendado la autorización bajo circunstancias excepcionales. 


\section{APREMILAST $\left(\text { OTEZL } A^{\circledR}\right)^{5-8}$}

Apremilast es un inhibidor oral selectivo de la fosfodiesterasa 4 (FDE4), que actúa intracelularmente modulando la red de mediadores proinflamatorios y antiinflamatorios. La inhibición de la FDE4 eleva los niveles de AMPc intracelular, manifestándose el bloqueo de la expresión de mediadores proinflamatorios como TNF-alfa, IL-23, IL-17 y otras citoquinas inflamatorias. Paralelamente aumenta la producción de mediadores antiinflamatorios como IL-10 o TFG beta, entre otros, lo que se traduce a nivel clínico en una reducción del nivel de inflamación en células de la piel y articulaciones.

Tiene dos indicaciones aprobadas: Artritis psoriásica: Se utiliza en pacientes adultos con artrititis psoriásica activa que hayan presentado intolerancia o respuesta inadecuada a una terapia con fármacos antirreumáticos modificadores de la enfermedad (FARME), siendo posible su uso en monoterapia o en combinación con otros FARME. Psoriasis: Indicado para el tratamiento de la psoriasis en placa crónica de moderada a grave en pacientes adultos en los que exista algún tipo de intolerancia, contraindicación o falta de respuesta a otras terapias sistémicas como ciclosporina, metotrexato o psoralenos con luz ultravioleta (PUVA).

Se ha comprobado seguridad, eficacia y tolerabilidad en un estudio denominado PALACE en el que apremilast ha mostrado una mejoría relevante de los signos y síntomas de la artritis psoriásica, según los criterios de mejoría ACR20 de la American College of Rheumatology, en comparación con placebo en la semana 16. Esta respuesta se mantuvo en la semana 24.

Apremilast mejora notoriamente la placa psoriásica de moderada a grave, objetivado por la proporción de pacientes con respuesta en el Índice de Gravedad y Área afectada de Psoriasis 75 (mejoría del PASI de al menos un 75\% respecto al valor basal) y en la Evaluación Global estática del Médico (sPGA static Physician Global Assessment) en la semana 16 comparada con placebo.

Las reacciones adversas más comunes observadas durante el desarrollo clínico fueron trastornos gastrointestinales incluyendo diarrea y náusea. Otras reacciones adversas comunes fueron infección del tracto respiratorio superior y cefalea.

El 20 de noviembre de 2014, el Comité de Medicamentos de Uso Humano de la Agencia Europea del Medicamento emitió una opinión positiva, recomendando la concesión de una autorización de comercialización del medicamento Otezla ${ }^{\circledR} 10$ mg, 20 mg y $30 \mathrm{mg}$.

\section{BALUGRASTIM $(\text { EGRANLI })^{\circledR-11}$}

Balugrastim es un medicamento inmunoestimulador que regula la producción y liberación de neutrófilos funcionales de la médula ósea. Se trata de una proteína obtenida por fusión genética de albumina humana con factor estimulante de colonias de granulocitos (G-CSF). Se presenta en forma de solución para inyección subcutánea de $40 \mathrm{mg}$, que se administra una vez en cada ciclo quimioterapéutico.

La indicación aprobada es la reducción de la duración de la neutropenia y de la incidencia de la neutropenia febril en pacientes adultos tratados con quimioterapia citotóxica para neoplasias malignas (con excepción de leucemia mieloide y síndromes mielodisplásicos).
La autorización se basa en dos estudios fase III, randomizados, doble ciegos, que incluyeron un total de 469 pacientes con cáncer de mama, asignadas al tratamiento con balugrastim $40 \mathrm{mg}(\mathrm{n}=235) \mathrm{o}$ pegfilgrastim $6 \mathrm{mg}(\mathrm{n}=234)$ cada $24 \mathrm{~h}$ tras administración de quimioterapia. La variable principal del estudio fue la duración de neutropenia grave (DNG) durante el primer ciclo. Ambos estudios mostraron no-inferioridad de balugrastim frente a pegfilgrastim. Las reacciones adversas más comunes observadas durante el desarrollo clínico fueron: dolor musculoesquelético, trombocitopenia, cefalea, leucocitosis, reacciones de la piel y aumento de enzimas hepáticas.

El 25 de septiembre de 2014, el CHMP emitió una opinión positiva, recomendando la concesión de la autorización de comercialización de Egranli®. Se recomienda su prescripción y supervisión por médicos con experiencia en oncología o hematología.

\section{DARUNAVIR/COBICISTAT ( REZOLSTA $\left.{ }^{\circledR}\right)^{9,12,13}$}

El principio activo es darunavir, un antirretroviral que actúa inhibiendo la actividad catalítica de la proteasa del VIH-1. Rezols$\mathrm{ta}^{\mathbb{R}}$ es una combinación a dosis fijas de darunavir y cobicistat, un potenciador farmacocinético que inhibe la isoenzima $3 \mathrm{~A}$ del citocromo P450 (CYP3A) y por lo tanto aumenta las concentraciones sanguíneas de darunavir. Se presenta en forma de comprimido recubierto con película de $800 \mathrm{mg} / 150 \mathrm{mg}$. La dosis recomendada es de un comprimido al día con comida.

Está indicado en combinación con otros antirretrovirales para el tratamiento de adultos mayores de 18 años, infectados por el virus de la inmunodeficiencia humana (VIH 1). Su uso se recomienda a los pacientes no tratados previamente con antirretrovirales (ARV), así como a los tratados anteriormente con estos medicamentos, siempre que se descarten mutaciones asociadas a resistencia a darunavir. Rezolsta ${ }^{\circledR}$ no se debe usar en pacientes con ARN del VIH-1 $\geq 100.000$ copias $/ \mathrm{ml}$ o con recuento de linfocitos CD4+ $<100$ células x 106/1.

Las conclusiones sobre la eficacia de Rezolsta ${ }^{\circledR}$ administrado una vez al día se basan principalmente en el análisis de los datos obtenidos en el ensayo GS-US-216-130 realizado con 313 pacientes con y sin tratamiento ARV (295 y 18, respectivamente). Se trata de un estudio fase III, de un solo brazo, abierto, multicéntrico, que evaluó la seguridad y la eficacia de un régimen de darunavir/ cobicistat ( $800 \mathrm{mg} / 150 \mathrm{mg}$ una vez al día con comida) más $2 \mathrm{ARV}$ plenamente activos en pacientes infectados por VIH-1. La variable principal de eficacia se definió como el porcentaje de pacientes con un nivel de ARN del VIH-1 $<50$ copias/ml a las 48 semanas. Esta combinación ha mostrado que proporciona una supresión virológica sostenible si se administra como parte de una combinación con otros medicamentos antirretrovirales. Las reacciones adversas más comunes observadas durante el desarrollo clínico fueron: diarrea, náuseas y erupción cutánea. Debido a las incertidumbres relacionadas con la velocidad del desarrollo de la barrera hematoencefálica humana y las enzimas hepáticas, no se debe usar en pacientes pediátricos menores de 3 años.

\section{DASABUVIR SÓDICO $\left(\text { EXVIER } A^{\circledR}\right)^{5,14-16}$}

Dasabuvir sódico es un inhibidor no análogo de nucleósido de la ARN polimerasa del ARN del VHC, codificada por el gen NS5B. 
Indicado en combinación con otros medicamentos para el tratamiento de la hepatitis $\mathrm{C}$ crónica en pacientes adultos.

Dasabuvir en combinación con otros medicamentos ha demostrado capacidad para inhibir la replicación viral en las células huésped infectadas. Esto puede conducir a la erradicación del virus y por tanto a una cura de la infección por virus de la hepatitis C crónica, tanto en pacientes no cirróticos como en pacientes cirróticos compensados con infección por VHC con genotipo 1a/1b.

Se ha estudiado su seguridad y eficacia fundamentalmente en seis estudios fase III (SAPPHIRE-I, SAPPHIRE-II, PEARL-II, PEARL-III, PEARL-IV y TURQUOISE-II) contando con la participación de unos 2.300 pacientes, todos ellos con genotipo 1 .

Destacando los siguientes, por haber mostrado eficacia, seguridad y tolerabilidad:

Estudio SAPPHIRE-I, aleatorizado, controlado con placebo y realizado en pacientes con genotipo 1 no tratados previamente y sin cirrosis. Se evaluó la eficacia del tratamiento combinado de ABT-450 (150 mg) y ombitasvir en una sola dosis diaria combinado con ritonavir (100mg), ombitasvir (25mg) y dasabuvir (250mg) 2 veces al día, además de ABT-333 y ribavirina ajustada al peso. Los pacientes fueron aleatorizados en 2 brazos de tratamiento: uno con la combinación de fármacos durante 12 semanas y otro con placebo. El objetivo primario era la obtención de una respuesta viral sostenida (RVS) en la semana 12 post tratamiento. El grupo de tratamiento fue comparado con la tasa de respuesta en un grupo de las mismas características tratados con triple terapia con telaprevir (grupo control). Se incluyeron 631 pacientes. La tasa de respuesta con el tratamiento fue de $96,2 \%$, el $95,3 \%$ en pacientes con genotipo 1a y un $98 \%$ en genotipo $1 \mathrm{~b}$, que fue superior al grupo control histórico con telaprevir. Un $0,2 \%$ sufrió un fracaso virológico durante el tratamiento y un 1,5\% recidivó tras el mismo. Los efectos secundarios fueron en general de grado leve y únicamente un $0,6 \%$ tuvo que abandonar el tratamiento.

Estudio SAPPHIRE II, aleatorizado, doble ciego y comparado con placebo fue diseñado para evaluar la eficacia y seguridad de la combinación terapeútica comentada para el estudio SAPPHIRE I. Se incluyeron pacientes con genotipo 1 , no cirróticos y se añadieron aquellos sin respuesta a tratamiento previo con peg-IFN y RBV (recidivantes, respondedores parciales, respondedores nulos). Se aleatorizaron en 2 brazos de tratamiento: una rama de placebo y otra de tratamiento activo durante 12 semanas, con un seguimiento hasta 48 semanas post tratamiento. La eficacia del tratamiento también se comparó con un grupo control con telaprevir en un estudio de no inferioridad. De los 297 pacientes que recibieron la combinación activa, el 98,7\% consiguió una respuesta viral en la semana 4 de tratamiento y el 99\% en la semana 12. Lograron una RVS12 el 95,3\% de los pacientes recidivantes, el 95,2\% de los respondedores nulos y el $100 \%$ de los respondedores parciales. Estos resultados muestran no inferioridad/superioridad de estos tratamientos en comparación con la triple terapia basada en telaprevir. El resultado fue independiente del genotipo. Tras finalizar tratamiento sólo 7 pacientes sufrieron una recidiva, identificándose variantes de resistencia. Los pacientes con un régimen activo tuvieron un descenso más marcado de ALT que en el grupo placebo de forma significativa. Los efectos adversos fueron leves, siendo los más frecuentes astenia, cefalea y náuseas.

El 20 de Noviembre de 2014 el Comité de Medicamentos de Uso Humano de la Agencia Europea del Medicamento adoptó una opinión positiva respecto al medicamento.

\section{DULAGLUTIDA (TRULICITY $\left.{ }^{\circledR}\right)^{9,17}$}

Dulaglutida es un agonista del receptor del péptido similar al glucagón de tipo 1 (GLP-1) generado por fusión de un análogo de GLP-1 a un fragmento de inmunoglobulina humana modificada (traduciéndose en prolongación de la vida media). Se comporta como GLP-1 nativa: mejora secreción de insulina dependiente de la glucosa y reduce la liberación de glucagón.

Está indicado en adultos con Diabetes Mellitus tipo 2 para mejorar el control glucémico, tanto en monoterapia como en combinación con otros antidiabéticos (inclusive insulina). Se utiliza en monoterapia en pacientes en los que no se consigue adecuado control glucémico con dieta y ejercicio, en los que se presente cierta intolerancia o contraindicación a metformina. Adicionándose en combinación a otros antidiabéticos orales (ADO), si éstos por sí solos no consiguen glucemias adecuadas.

La seguridad y eficacia fueron evaluadas a través de seis ensayos clínicos de fase III aleatorizados, controlados, en 5.171 pacientes con diabetes tipo 2 . Se estudió el comportamiento de dulaglutida en monoterapia cuando la metformina no puede utilizarse y en combinación con otros antidiabéticos incluyendo insulina. Los parámetros evaluados fueron la disminución de hemoglobina glicosilada A1c y el tiempo en alcanzar los niveles objetivo de HbA1c de $<7,0 \% \mathrm{y} \leq 6,5 \%$. También se han descrito para cada uno de los ensayos las tasas de hipoglucemia sintomática, obteniéndose en total 12 casos de hipoglucemias de carácter grave en 2 de los estudios.

En cuanto a resultados, se ha observado un efecto clínicamente relevante sobre el control glucémico además de cierto beneficio en relación al control del peso corporal.

Durante el desarrollo clínico la reacciones adversas más comunes fueron la hipoglucemia (que sólo se presentó al usar la dulaglutida con otros antidiabéticos orales), náuseas y diarrea.

El 21 de noviembre de 2014 el Comité de Medicamentos de Uso Humano de la Agencia Europea del Medicamento adoptó una opinión positiva al respecto del medicamento.

\section{ELIGLUSTAT (CERDELGA $\left.{ }^{\circledR}\right)^{5,18-20}$}

Eliglustat es un inhibidor de la glucosilceramida sintasa que actúa reduciendo la producción de glucosilceramida, la cual se deposita en los tejidos en personas con la enfermedad de Gaucher tipo 1. También disminuye los niveles plasmáticos de glucosilceramida.

Está indicado para el tratamiento a largo plazo de pacientes adultos con enfermedad de Gaucher tipo 1 que sean metabolizadores lentos, intermedios o rápidos del CYP2D6.

La eficacia y seguridad ha sido evaluada en un estudio en fase II, multicéntrico, abierto y de un único brazo de tratamiento. Se incluyeron 26 pacientes con esplenomegalia, trombocitopenia y/o anemia, de los cuales completaron las 52 semanas 22 de ellos. El estudio mostró la disminución de los niveles plasmáticos de glucosilceramida, mejorando el volumen de órganos (bazo: - 38,5\% ; IC del $95 \%=-43,5 \%-33,5 \%$ e hígado: $-17,0 \%$; IC del $95 \%=-21,6 \%$ $-12,3 \%$ ), parámetros hematológicos (niveles de hemoglobina: 1,62 $\mathrm{g} / \mathrm{dl}$; IC del $95 \%=1.5$ a $2.18 \mathrm{~g} / \mathrm{dL}$ y recuento de plaquetas: $40.3 \%$, IC del $95 \%=23,7-57,0 \mathrm{~g} / \mathrm{dL}$ ) y esqueléticos (densidad mineral ósea: 0.31 Z-score; IC del 95\% = 0,09-0,53). 
Durante el desarrollo clínico las reacciones adversas observadas con mayor frecuencia fueron: cefalea, náuseas, diarrea, dolor abdominal, flatulencia, artralgia y fatiga.

Cerdelga $^{\circledR}$ fue designado como medicamento huérfano el 4 de diciembre de 2007, el 20 de Noviembre de 2014 el Comité de Medicamentos de Uso Humano de la Agencia Europea del Medicamento adoptó una opinión positiva respecto al medicamento.

\section{ESTRÓGENOS CONJUGADOS/BAZEDOXIFENO $\left(\boldsymbol{D} \boldsymbol{U} \boldsymbol{A} \boldsymbol{V I V} \boldsymbol{E}^{\circledR}\right)^{1,21,22}$}

Los principios activos son estrógenos conjugados y bazedoxifeno. Los estrógenos conjugados se unen a los receptores $\alpha$ y $\beta$ de estrógeno y los activan, sustituyendo de esa manera la pérdida de estrógenos en las mujeres menopáusicas y aliviando los síntomas de la menopausia. El riesgo aumentado de hiperplasia endometrial y cáncer asociado al uso de estrógenos se reduce al añadir bazedoxifeno, que actúa como un antagonista de los receptores de estrógenos en el útero.

Duavive $^{\circledR}$ se presenta en forma de comprimidos de liberación modificada $(0.45 \mathrm{mg} / 20 \mathrm{mg})$.

La indicación aprobada es tratamiento de los síntomas asociados a la deficiencia de estrógenos en mujeres posmenopáusicas que conservan el útero (y en las que hayan transcurrido al menos 12 meses desde la última menstruación) para las que el tratamiento con progestágenos no es apropiado. La experiencia es limitada en el tratamiento de mujeres mayores de 65 años.

La seguridad y la eficacia de Duavive ${ }^{\circledR}$ como tratamiento de síntomas vasomotores asociados con la menopausia se han establecido principalmente en un estudio fase III, doble ciego, controlado con placebo, de 12 semanas de duración. El estudio ha incluido un total de 3.397 mujeres (edad de 40 a 75 años) que presentaban al menos 7 sofocos de intensidad moderada a grave por día o por lo menos 50 sofocos por semana. A la semana 12, el numero diario de sofocos disminuyó $85.7 \%$ con BZA/CE y $17.1 \%$ con placebo. La reacción adversa más común observada durante el desarrollo clínico fue dolor abdominal, identificado en más del $10 \%$ de los sujetos.

E1 23 de octubre de 2014, el CHMP emitió una opinión positiva, recomendando la concesión de la autorización de comercialización de Duavive ${ }^{\circledR}$.

\section{KETOCONAZOL (KETOCONAZOL HRA PHARMA $\left.{ }^{\circledR}\right)^{9,24}$}

Ketoconazol es un potente inhibidor de la síntesis del cortisol, aldosterona y andrógenos, debido a su capacidad de inhibir enzimas del citocromo P450 en las glándulas suprarrenales. Aparte del efecto de bloqueo adrenal, ketoconazol también puede tener efectos directos sobre las células tumorales corticotrópicas en pacientes con enfermedad de Cushing.

Se presenta en forma de comprimidos de $200 \mathrm{mg}$. Ketoconazol vía oral ha estado autorizado durante décadas en varios países de la Unión Europea para el tratamiento de infecciones fúngicas, aunque se ha utilizado también fuera de indicación en el manejo del síndrome de Cushing.

La indicación aprobada es el tratamiento del síndrome de Cushing endógeno en adultos y adolescentes mayores de 12 años. En los ensayos clínicos este medicamento ha mostrado que controla el hipercortisolismo a largo plazo. La mejoría bioquímica y hormonal que se observa (descenso o normalización de los niveles urinarios de cortisol libre) se asocia a mejoría clínica de los síntomas del síndrome de Cushing.

Las reacciones adversas más comunes observadas durante el desarrollo clínico fueron: insuficiencia adrenal, náuseas, vómitos, dolor abdominal, diarrea, prurito, erupciones e incremento de las enzimas hepáticas. La reacción adversa más importante desde el punto de vista clínico es la hepatotoxicidad que obliga a monitorizar la función hepática antes y durante el tratamiento. Debe monitorizarse también la función adrenal y los cambios en el electrocardiograma. Además, se debe valorar cuidadosamente el potencial de interacciones fármaco-fármaco, que pueden producirse como consecuencia de su metabolismo. La dosis se debe ajustar de acuerdo a los niveles de cortisol y con el fin de normalizar sus niveles.

Ketoconazol HRA Pharma ${ }^{\circledR}$ fue designado como medicamento huérfano para el tratamiento de síndrome de Cushing el 23 de abril de 2012 y presentado posteriormente por la compañía para registro.

\section{NALOXEGOL (MOVENTIG $\left.{ }^{\circledR}\right)^{9,25,26}$}

Se trata de un derivado pegilado de naloxona que actúa como antagonista de los receptores opioides $\mu$ periféricos, sobre todo en el sistema gastrointestinal. Debido a su estructura química, disminuye el estreñimiento inducido por opiáceos sin afectar sus efectos analgésicos sobre el sistema nervioso central. Esto es debido al hecho que la pegilación reduce la permeabilidad pasiva de naloxegol que se vuelve sustrato para la glicoproteína $\mathrm{P}$, lo que aumenta su flujo a través de la barrera hematoencefálica.

Se presenta en forma de comprimidos de $12.5 \mathrm{mg}$ y $25 \mathrm{mg}$. Está indicado para tratamiento del estreñimiento inducido por opiáceos en pacientes adultos que no responden adecuadamente a laxantes.

La eficacia de naloxegol se mostró en dos estudios fase III pivotales, de 12 semanas de duración, multicéntricos, aleatorizados, doble ciegos, controlados con placebo, que incluyeron 1352 sujetos y evaluaron las dosis de $12.5 \mathrm{mg}$ y de $25 \mathrm{mg}$ de naloxegol administrado una vez al día. Este medicamento ha mostrado producir tasas de respuesta más altas que el placebo en la variable principal de eficacia, definida como más de 3 movimientos espontáneos del intestino por semana y un cambio desde valores basales de más de un movimiento espontáneo del intestino por semana, durante al menos 9 de las 12 semanas del estudio y en 3 de las 4 últimas semanas.

Las reacciones adversas más comunes observadas durante el desarrollo clínico fueron: dolor abdominal, diarrea, náuseas, cefalea y flatulencia. La mayoría de los efectos adversos gastrointestinales fueron leves a moderados, se produjeron al principio del tratamiento y se resolvieron con administración continuada del medicamento.

\section{NINTEDANIB (VARGATEF $\left.F^{\circledR} / O F E V^{\circledR}\right)^{5,9,27-30}$}

Este principio activo ha sido aprobado para dos indicaciones diferentes con un nombre comercial distinto para cada una de ellas. 


\section{Evaluación positiva de medicamentos: septiembre/octubre/noviembre 2014}

\section{VARGATEF ${ }^{\circledR}$}

Nintedanib es un triple inhibidor de la tirosina quinasa que actúa a nivel de los receptores del factor de crecimiento endotelial vascular (VEGFR 1-3), los receptores de factores de crecimiento derivados de plaquetas (PDGFR $\alpha$ y $\beta$ ) y los receptores del factor de crecimiento de fibroblastos (FGFR 1-3), produciéndose inhibición de la angiogénesis tumoral.

Se ha aprobado su indicación como Vargatef ${ }^{\circledR}$ en combinación con docetaxel para el tratamiento de pacientes adultos con adenocarcinoma de pulmón no microcítico (CPNM), localmente avanzado, metastásico o localmente recidivante después de la primera línea de quimioterapia.

La eficacia y la seguridad de $\operatorname{Vargatef}^{\circledR}$ se ha evaluado mediante un ensayo de fase III (LUME-lung1) donde se evaluaron 1.314 pacientes adultos con CPNM localmente avanzado, metastásico o recurrente después de una primera línea de quimioterapia. El ensayo incluyó a 658 pacientes con adenocarcinoma, 555 pacientes con carcinoma epidermoide y 101 pacientes con otras histologías tumorales. Los pacientes se aleatorizaron (1:1) para recibir nintedanib $200 \mathrm{mg}$ por vía oral dos veces al día en combinación con 75 $\mathrm{mg} / \mathrm{m}^{2}$ de docetaxel intravenoso cada 21 días $(\mathrm{n}=655)$ o placebo por vía oral dos veces al día en combinación con $75 \mathrm{mg} / \mathrm{m}^{2}$ de docetaxel cada 21 días $(\mathrm{n}=659)$.

La variable principal del estudio fue la supervivencia libre de progresión, siendo también estudiada la supervivencia global.

Nintedanib asociado a docetaxel produjo una reducción estadísticamente significativa del $21 \%$ en el riesgo de progresión o muerte (hazard ratio 0,79; IC de $95 \%$ : 0,68-0,92; $\mathrm{p}=0,0019$ ), y una hazard ratio de 0,85; IC del $95 \%$ : 0,75-0,96; $p=0,0070$ en la variable supervivencia global. El análisis de la supervivencia global no alcanzó una significación estadística. Sin embargo en el análisis según la histología, entre los grupos de tratamiento, se observó diferencia estadísticamente signficativa para adenocarcinoma.

Entre las reacciones adversas observadas con mayor frecuencia durante el desarrollo clínico se encuentran: neutropenia (incluyendo neutropenia febril), anorexia, alteraciones electrolíticas, neuropatía periférica, sangrados, diarrea, vómitos, náuseas, aumento de transaminasas, mucositis (incluyendo estomatitis) y erupción cutánea.

El nintedanib fue designado medicamento huérfano el 26 de abril de 2013, siendo el 25 de septiembre de 2014 emitida una opinión positiva por parte del Comité de Medicamentos de Uso Humano de la Agencia Europea del Medicamento al respecto del medicamento.

\section{OFEV $^{\circledR}$}

Por el mismo mecanismo de acción descrito arriba, nintedanib resulta también crucial para la proliferación y migración de los fibroblastos pulmonares, lo que se traduce en una disminución del proceso desencadenante de la fibrosis pulmonar.

Ofev $^{\circledR}$ está indicado para el tratamiento de la fibrosis pulmonar idiopática en adultos, la dosis recomendada es $150 \mathrm{mg}$.

La eficacia y seguridad han sido evaluadas en dos estudios pivotales de fase III, aleatorizados, doble ciego, controlados con placebo y grupos paralelos. INPULSIS-1 e INPULSIS-2, incluyeron 513 y 548 pacientes respectivamente, siendo criterios de inclusión tener 40 años o más y haber sido diagnosticados de fibrosis pulmonar idiopática en los 5 años previos. En ellos se ha observado que Ofev ${ }^{\circledR}$ reduce la tasa de deterioro de la función pulmonar, medida como la reducción del volumen absoluto de la capacidad vital forzada (CVF). Ambos estudios mostraron un beneficio claro y consistente sobre la disminución del declive de la CVF en aproximadamente $94 \mathrm{ml} /$ año y $125 \mathrm{ml} /$ año respectivamente.

Las reacciones adversas más comunes observadas durante el desarrollo clínico fueron: trastornos gastrointestinales, diarrea, vómitos, náuseas, y aumento de las transaminasas.

El 26 de abril fue designado como medicamento huérfano y el 20 de Noviembre de 2014 el Comité de Medicamentos de Uso Humano de la Agencia Europea del Medicamento adoptó una opinión positiva respecto a $\mathrm{Ofev}^{\circledR}$.

\section{NONACOG GAMMA $\left(\boldsymbol{R I X U B I S ^ { \circledR }}\right)^{1,31,32}$}

La hemofilia B es una enfermedad congénita ligada al cromosoma X que se caracteriza por la disminución o ausencia total de factor IX de coagulación. Eso resulta en hemorragias en articulaciones, músculos u órganos internos, espontáneas o como consecuencia de trauma accidental o quirúrgico. Terapia de sustitución de factor IX proporciona una corrección temporal de la deficiencia de este factor, corrigiendo así la tendencia al sangrado.

El principio activo es nonacog gamma, factor IX de coagulación recombinante. Éste una vez activado, en combinación con el factor VIII activado, activa el factor X que convierte la protrombina en trombina. La trombina convierte el fibrinógeno en fibrina produciéndose la consiguiente formación del coágulo.

Está disponible como polvo liofilizado en viales de 250, 500, 1000,2000 y 3000 unidades internacionales (UI). Una UI de Rixubis ${ }^{\circledR}$ por kilogramo del peso corporal aumenta la actividad circulante del factor IX por $0.7 \mathrm{UI} / \mathrm{dL}$ en pacientes menores de 12 años y por $0.9 \mathrm{UI} / \mathrm{dL}$ en pacientes mayores de esta edad. La indicación aprobada es el tratamiento y profilaxis de hemorragias en pacientes con hemofilia $\mathrm{B}$ de todas las edades.

La eficacia y la seguridad de Rixubis ${ }^{\circledR}$ en pacientes menores de 12 años se ha mostrado en un ensayo clínico fase II/III no controlado que incluyo 23 pacientes masculinos con hemofilia B previamente tratados. Los pacientes fueron tratados con Rixubis ${ }^{\circledR}$ en régimen profiláctico bisemanal (dosis mediana $56 \mathrm{IU} / \mathrm{kg}$ ) durante al menos tres meses. Nueve pacientes en el estudio (39.1\%) no experimentaron sangrado y 14 pacientes $(88,5 \%)$ con 26 episodios de sangrado) fueron tratados con 1 o 2 infusiones. En pacientes mayores de 12 años, la eficacia ha sido evaluada en un ensayo multicéntrico prospectivo, abierto, no controlado, en el cual un total de 59 varones recibieron Rixubis ${ }^{\circledR}$ como profilaxis rutinaria durante al menos 3 meses en dosis bisemanal de 40 a $60 \mathrm{UI} / \mathrm{kg}$. La media de cualquier sangrado fue 4.3 en el grupo con la profilaxis activa comparando con una media de 20 en el grupo de control histórico. En ambos estudios, infusión de Rixubis ${ }^{\circledR}$ resolvió 96\% de todos los episodios de sangrado. Las reacciones adversas más comunes observadas durante el desarrollo clínico fueron: disgeusia, dolor en las extremidades, formación de anticuerpos anti-Furin y anemia postoperatoria.

El 23 de octubre de 2014, el CHMP emitió una opinión positiva, recomendando la concesión de la autorización de comercialización de Rixubis ${ }^{\circledR}$. 


\section{OLAPARIB ( LYNPARZA $\left.A^{\circledR}\right)^{1,33,34}$}

Se trata de un inhibidor de la poli ADP-ribosa polimerasa humana (PARP-1, PARP-2, y PARP-3), necesaria para la reparación de la cadena sencilla de ADN.

La rotura de la cadena de ADN en las células normales también se puede reparar mediante un proceso conocido como reparación homóloga de recombinación (RHR), que requiere los genes BRCA1 y BRCA2 funcionales. Sin embargo, en las células cancerígenas sin genes BRCA1 o BRCA2 funcionales, las roturas de la cadena sencilla no pueden ser reparados a través de RHR, haciéndolas vulnerables a la inhibición de PARP por olaparib. Se presenta en capsulas duras de $50 \mathrm{mg}$, siendo la dosis diaria 400mg.

Esta indicado en monoterapia para el tratamiento de mantenimiento en pacientes adultos con cáncer de ovario seroso de alto grado, cáncer de la trompa de Falopio o peritoneal primario en recaída, que presentan mutación del BRCA (germinal y/o somática), y que responden (res-puesta completa o parcial) a quimioterapia basada en platino. Hasta ahora, no existía un tratamiento de mantenimiento indicado para las pacientes que han sufrido la recaída de la enfermedad tras la respuesta inicial a platino.

Lynparza ${ }^{\circledR}$ fue designado como medicamento huérfano el 6 de diciembre de 2007; el 23 de octubre de 2014, el CHMP emitió una opinión positiva, recomendando la concesión de la autorización de comercialización de olaparib.

La autorización se basa en un ensayo clínico fase II que evaluó la eficacia y la seguridad de olaparib comparado con placebo en 265 pacientes con cáncer de ovário seroso de alto grado, recidivante, sensible a platino. El estudio mostró que la terapia de mantenimiento con olaparib prolonga significativamente la supervivencia libre de progresión en comparación con el placebo en pacientes con mutación BRCA-11,2 meses frente a 4,3 meses (HR = 0.18; IC $95 \%, 0.10-0,31 ; \mathrm{p}<0.0001)$. Las reacciones adversas más comunes observadas durante el desarrollo clínico fueron: náuseas, vómitos, diarrea, dispepsia, fatiga, dolor de cabeza, disgeusia, disminución del apetito, mareos, anemia, neutropenia, linfopenia, elevación del volumen corpuscular, y aumento de la creatinina.

\section{OMBITASVIR, PARITAPREVIR, RITONAVIR $\left(\text { VIEKIRAX } \boldsymbol{X}^{\circledR}\right)^{5,35}$}

Este medicamento es la asociación de varios principios activos: a) ombitasvir, un inhibidor de la proteína no estructural NS5A del VHC, que es esencial para la replicación viral; b) paritaprevir, un inhibidor de la proteína no estructural proteasa NS3/4A, también esencial para la replicación viral; y c) ritonavir, un potente inhibidor del citocromo P450 3A4 usado como potenciador farmacocinético.

La indicación aprobada es, en combinación con otros medicamentos, el tratamiento de la hepatitis $\mathrm{C}$ crónica en pacientes adultos. $\mathrm{Y}$ debe ser prescrito por un médico especialista en «hepatitis $\mathrm{C}$ crónica».

El beneficio aportado por este medicamento en combinación con otros es la capacidad para inhibir la replicación viral en células huésped infectadas, tanto en pacientes con cirrosis compensada como en no cirróticos (genotipo 1A/1b y 4 ).

Las reacciones adversas más comunes observadas durante el desarrollo clínico fueron fatiga y náuseas. Un plan de farmacovigilancia será una exigencia tras la comercialización del medicamento.
Las recomendaciones para su uso se describirán en la ficha técnica del producto, que estará disponible tras la autorización de comercialización por parte de la Comisión Europea, normalmente se emite 67 días a partir de la adopción de la opinión positiva del «Committee for Medicinal Products for Human Use» de la Agencia Europea del Medicamento, que tuvo lugar el 20 de noviembre de 2014.

\section{OSPEMIFENO (SENSHIO $\left.{ }^{\circledR}\right)^{5,36,37}$}

Ospemifeno se comporta como modulador (agonista/antagonista) selectivo del receptor de estrógenos, activando vías estrogénicas en algunos tejidos y bloqueándolas en otros tejidos. Es el metabolito principal del toremifeno, que como el tamoxifeno también es utilizado en el cáncer de mama.

Tras más de 20 años en desarrollo el ospemifeno fue aprobado por la Food and Drugs Administration en 2013 , con el nombre comercial de Osphena ${ }^{\circledR}$ y con la la misma indicación recientemente aprobada por la Agencia Europea del Medicamento.

La indicación aprobada es en el tratamiento de la atrofia vulvovaginal sintomática de moderada a grave en mujeres postmenopáusicas no candidatas a terapia local vaginal con estrógenos.

Su eficacia, seguridad y tolerabilidad se ha estudiado en 6 ensayos clínicos fase III. Se ha observado como ospemifeno $60 \mathrm{mg}$ respecto a $30 \mathrm{mg}$, mejoró significativamente la mayor parte de las variables clínicas estudiadas, comparado con placebo. Se ha obtenido resultado positivos en parámetros como el índice de maduración vaginal (disminución de las células parabasales y aumento de células superficiales), disminución del $\mathrm{pH}$ vaginal y disminución de la sensación de molestia como dispareunia o sequedad vaginal.

En cuanto a seguridad y tolerabilidad, los ensayos revelaron que dosis de $60 \mathrm{mg}$ de ospemifeno administrado diariamente durante 52 semanas fueron óptimas, no asociando afectación endometrial o problemas de seguridad relacionados con la mama.

Durante el desarrollo clínico la reacción adversa más frecuentemente observada fue el sofoco.

El 20 de noviembre de 2014 el Comité de Medicamentos de Uso Humano de la Agencia Europea del Medicamento adoptó una opinión positiva respecto al medicamento.

\section{RAMUCIRUMAB ( $\left.C Y R A M Z A^{\circledR}\right)^{9,38}$}

Se trata de un anticuerpo humano antagonista que se une específicamente al receptor 2 del factor de crecimiento del endotelio vascular VEGF-A, VEGF-C y VEGF-D, bloqueando la angiogénesis.

Está indicado para el tratamiento de pacientes adultos con cáncer gástrico avanzado o adenocarcinoma de la unión gastroesofágica con progresión de la enfermedad tras quimioterapia previa con platino y fluoropirimidina. Se utiliza en combinación con paclitaxel o como monoterapia en pacientes para quienes el tratamiento con paclitaxel no es apropiado.

Se presenta en forma de solución para inyección de $10 \mathrm{mg} /$ $\mathrm{ml}$. La pauta recomendada es de $8 \mathrm{mg} / \mathrm{kg}$ cada dos semanas, en infusión de 60 minutos.

La autorización se basa en un estudio fase III, multicéntrico, randomizado, doble ciego que ha incluido 665 pacientes con cáncer gástrico avanzado o adenocarcinoma de la unión gas- 
troesofágica con progresión de la enfermedad tras quimioterapia previa con platino y fluoropirimidina. El primer grupo de pacientes $(\mathrm{n}=330)$ fue tratado con Cyramza ${ }^{\circledR} 8 \mathrm{mg} / \mathrm{kg}$ (días $1 \mathrm{y}$ 15 de ciclo de 28 días) y paclitaxel $80 \mathrm{mg} / \mathrm{m}^{2}$ (días 1,8 y 15 de ciclo de 28 días), mientras el grupo control $(\mathrm{n}=335)$ tenía la misma pauta de paclitaxel (días 1, 8 y 15) asociada a placebo los días 1 y 15. Los pacientes recibieron tratamiento hasta la progresión de la enfermedad o la toxicidad inaceptable. La medida principal de la eficacia fue la supervivencia global que ha mejorado significativamente en los pacientes que recibieron Cyramza ${ }^{\circledR}$ en comparación con los pacientes asignados al placebo.

Las reacciones adversas más comunes observadas durante el desarrollo clínico fueron: fatiga, astenia, neutropenia, leucopenia, diarrea, epistaxis e hipertensión.

Se recomienda su prescripción y supervisión por médicos con experiencia en la administración de terapia anticancerígena.

\section{SECUKINUMAB (CONSENTY $\left.X^{\circledR}\right)^{5,39-41}$}

Secukinumab es un anticuerpo monoclonal humano, se une selectivamente a la interleukina 17A neutralizándola ,impidiendo su unión con el receptor IL-17 expresado en varios tipos de células, incluidos los queratinocitos. De esta forma se inhibe la liberación de citoquinas proinflamatorias, quimioquinas y mediadores del daño tisular en enfermedades autoinmunes e inflamatorias como la psoriasis.

La indicación aprobada es para el tratamiento de la psoriasis en placa de moderada a grave en adultos que son candidatos a tratamiento sistémico. La dosis recomendada es de $300 \mathrm{mg} /$ día.

La eficacia, seguridad y tolerabilidad de secukinumab se ha estudiado a partir de dos ensayos pivotales ambos de fase III, multicéntricos, doble ciego y de 52 semanas de duración. Se comparó secukinumab versus placebo, en dos dosis diferentes (150 mg y 300mg subcutáneo), 1 vez por semana durante 5 semanas y posteriormente cada 4 semanas, en el denominado ensayo ESURE, con $n=738$. En el ensayo FIXTURE, con $n=1.306 \mathrm{se}$ comparó el secukinumab frente a etarnecept $50 \mathrm{mg} 2$ veces por semana durante 12 semanas y posteriormente 1 vez por semana.

El objetivo de ambos estudios fue demostrar la superioridad de secukinumab sobre el placebo y etarnecept en la semana 12.Los parámetros estudiados de mayor relevancia fueron el Índice de Gravedad y Área afectada de Psoriasis (PASI) y la Evaluación Global del Investigador (IGA).

El PASI mide el enrojecimiento, la descamación y el grosor de las placas de psoriasis, así como el alcance de la afectación de cada una de las partes del cuerpo. La eficacia del tratamiento se evalúa mediante la reducción de la puntuación respecto a los niveles basales (una reducción del 75\% se conoce como PASI 75, una reducción del 90\% se considera PASI 90, y PASI 100 representa el blanqueamiento total de la piel).

Ambos estudios han mostrado la superioridad de secukinumab tanto frente a placebo como a etanercept en la semana 12.

Durante el desarrollo clínico las reacciones adversas más comunes observadas fueron infecciones en el tracto respiratorio superior, siendo las más frecuentes nasofaringitis y rinitis.

El 20 de noviembre de 2014 el Comité de Medicamentos de Uso Humano de la Agencia Europea del Medicamento adoptó una opinión positiva respecto al medicamento4.

\section{SOFOSBUVIR/LEDIPASVIR (HARVONI $\left.{ }^{\circledR}\right)^{9,42-44}$}

Los principios activos son ledipasvir y sofosbuvir, una combinación a dosis fija de dos antivirales activos directos. El metabolito activo de sofosbuvir es un inhibidor pangenotípico de la NS5B RNA polimerasa del virus de la hepatitis C mientras el ledipasvir tiene como diana la proteína NS5A del virus.

Se presenta en forma de comprimidos que contienen $400 \mathrm{mg}$ de sofosbuvir y $90 \mathrm{mg}$ de ledipasvir, siendo la dosis recomendada un comprimido al día. La duración del tratamiento es de 12 semanas en los pacientes sin cirrosis y en los pacientes con cirrosis no tratados previamente. En los enfermos con cirrosis previamente tratados, el tratamiento debe durar 24 semanas.

La indicación aprobada es el tratamiento de la hepatitis C crónica genotipo 1 en adultos.

La eficacia de esta combinación se evaluó en tres ensayos clínicos fase III, que incluyeron 1518 sujetos con la enfermedad hepática compensada (con o sin cirrosis). Con o sin rivabirina, Harvoni ${ }^{\circledR}$ ha mostrado tener una eficacia alta contra los genotipos 1,3 y 4 , incluyendo pacientes post-trasplante y/ o con cirrosis compensada. Las reacciones adversas más comunes observadas durante el desarrollo clínico fueron fatiga y cefalea.

Se recomienda su prescripción y supervisión por médicos con experiencia en el tratamiento de la hepatitis $\mathrm{C}$ crónica en adultos.

\section{TILMANOCEPT ( LYMPHOSEEK $\left.{ }^{\circledR}\right)^{9,45-47}$}

Tilmanocept es un radiofármaco para uso diagnóstico. Contiene un componente de manosa que tiene una alta afinidad para proteínas receptoras conocidas como CD-206, que se encuentran en altas concentraciones en la superficie de los macrófagos y células dendríticas. Uniéndose a esos receptores, tilmanocept se acumula en el tejido linfático de los ganglios centinela.

Está indicado para el mapeo linfático (la obtención de imágenes y la detección intraoperatoria) de ganglios linfáticos centinela que drenan de un tumor primario en pacientes adultos con cáncer de mama, melanoma, o carcinoma epidermoide localizado en la cavidad oral, usando un dispositivo de detección gamma. En los estudios clínicos, Lymphoseek ${ }^{\circledR}$ ha sido detectable en los ganglios linfáticos a los 10 minutos tras inyección, permaneciendo en el tejido hasta 30 horas después. El mapeo linfático debe realizarse desde 15 minutos hasta las 15 horas post-inyección.

La eficacia de Lymphoseek ${ }^{\circledR}$ se mostró en dos ensayos pivotales fase III, abiertos, multicéntricos en los que a un total de 332 pacientes con melanoma o cáncer de mama (sin enfermedad nodal o metastásica) se les administró Lymphoseek ${ }^{\circledR}$ o los colorantes azules para la detección in vivo de los ganglios linfáticos centinela. Lymphoseek ${ }^{\circledR}$ mostró alta concordancia con los colorantes azules, siendo presente en el 97\% (rango 94\% - 100\%) de los ganglios linfáticos resecados.

En otro estudio, en pacientes con carcinoma epidermoide de cavidad oral se evaluó la capacidad de Lymphoseek ${ }^{\circledR}$ para detectar ganglios linfáticos positivos; histológicamente se encontró que el fármaco tiene una tasa de falsos negativos del 2,5\%.

Las reacciones adversas más frecuentes eran irritación en el sitio de la inyección y dolor $(<1 \%)$. No se observaron reacciones de hipersensibilidad graves. 
El 25 de septiembre de 2014, el CHMP emitió una opinión positiva, recomendando la concesión de la autorización de comercialización de Lymphoseek ${ }^{\circledR}$.

\section{VORAPAXAR (ZONTIVITY $\left.{ }^{\circledR}\right)^{5,48-50}$}

El principio activo es vorapaxar, un inhibidor selectivo y reversible del receptor I activado de proteasa presente en las plaquetas. Vorapaxar inhibe la agregación plaquetaria inducida por trombina in vitro e inhibe la agregación plaquetaria inducida por el péptido agonista del receptor de trombina, sin afectar los parámetros de coagulación.

Vorapaxar no inhibe la agregación plaquetaria inducida por otros agonistas como adenosina difosfato, colágeno o un mimético del tromboxano.

La indicación aprobada es la siguiente: «Zontivity, coadministrado con ácido acetilsalicílico, y cuando sea apropiado con clopidogrel, está indicado para la reducción de eventos aterotrombóticos en pacientes adultos con antecedentes de infarto de miocardio».

Las reacciones adversas más comunes observadas durante el desarrollo clínico estuvieron relacionadas con hemorragias, como hematomas, epistaxis o hematuria. Un plan de farmacovigilancia será una exigencia tras la comercialización del medicamento.

Respecto a este medicamento, señalamos que se interrumpió un ensayo clínico, aleatorizado, doble ciego, multicéntrico que comparaba vorapaxar con placebo en pacientes con síndrome coronario agudo sin elevación del ST $(n=12.944)$ por motivos de seguridad. La variable principal del estudio era una variable compuesta (muerte por cualquier causa o infarto de miocardio o accidente cerbrovascular o isquemia recurrente con rehospitalización o revasularización coronaria urgente). La proporción de sangrados en el grupo activo fue 7,2\% versus 5,2\% en el grupo placebo (hazard ratio: 1,35 IC 95\%: 1.16 a $1.58 ; \mathrm{p}<0.001$ ) y la hemorragia intracraneal también fue más frecuente en el grupo activo $(1.1 \%$ versus $0.2 \%$; hazard ratio: 3,39 IC 95\%: 1.78 a 6.45 ; $p<0.001)$.

Del mismo modo, en otro EC aleatorizado, doble ciego, se comparó este medicamento con placebo en 26.449 pacientes con historia de infarto agudo de miocardio, accidente cerebrovascular isquémico o enfermedad arterial periférica. El objetivo principal del estudio también era una variable compuesta (muerte de origen cardiovascular o infarto de miocardio o accidente cerebrovascular) y también se interrumpió por motivos de seguridad. La variable de eficacia fue favorable al grupo activo (hazard ratio: 0.88 IC 95\%: 0.82 a 0.95; $\mathrm{p}<0.001)$ pero también el mayor número de sangrados clasificados como de intensidad «moderada o grave» (hazard ratio: 1.66 IC 95\%: 1.43 a $1.93 ; \mathrm{p}<0.001$ ) y la proporción de hemorragias intracraneales $(1.0 \%$ versus $0.5 \%$; $<<0.001)$.

\section{BIBLIOGRAFÍA}

1. Agencia Española de Medicamentos y Productos Sanitarios. Informe mensual sobre Medicamentos de Uso Humano y Productos Sanitarios Octubre 2014. Disponible en: http://www.aemps.gob.es/informa/boletinMensual/2014/octubre/boletin-octubre.htm\#nuevosMed

2. Scenesse®. Disponible en: http://www.clinuvel.com/en/scenesse/scenesseafamelanotide

3. Clinuvel Pharmaceuticals Limited: »Phase III Confirmatory Study in Erythropoietic Protoporphyria». Disponible en: https:/clinicaltrials.gov/ct2/show/ NCT01605136
4. Committee for Medicinal Products for Human Use (CHMP). Afamelatonide. Disponible en: http://www.ema.europa.eu/ema/index.jsp?curl=pages/ medicines/human/medicines/002548/smops/Positive/human_smop_000747. jsp\&mid=WC0b01ac058001d127

5. Agencia Española de Medicamentos y Productos Sanitarios. Informe mensual sobre Medicamentos de Uso Humano y Productos Sanitarios. Noviembre 2014. Disponible en: http://www.aemps.gob.es/informa/boletinMensual/2014/ noviembre/docs/boletin-mensual_noviembre-2014.pdf

6. Arthur K, Philip J M,Juan JG, Adewale O A, Jürgen W, Dafna D et al. Treatment of psoriatic arthritis in a phase 3 randomised, placebo-controlled trial with apremilast, an oral phosphodiesterase 4 inhibitor. Ann Rheum Dis. 2014;73(6):1020-1026.Published online Mar 4, 2014. doi: 10.1136/annrheumdis-2013-205056 PMCID: PMC4033106. Disponible en: http://www.ncbi.nlm. nih.gov/pmc/articles/PMC4033106/

7. Mazur M, Karczewski J, Lodyga M, \aba R, Adamski Z. Inhibitors of phosphodiesterase 4 (PDE 4): A new therapeutic option in the treatment of psoriasis vulgaris and psoriatic arthritis. J Dermatolog Treat. 2014;29:1-3. (ABSTRACT) Disponible en: http://www.ncbi.nlm.nih.gov/pubmed/25424050

8. Committe for Medicinal Products for Human Use (CHMP). Disponible en: http://www.ema.europa.eu/docs/en_GB/document_library/Summary_of_opinion_-_Initial_authorisation/human/003746/WC500177621.pdf

9. Agencia Española de Medicamentos y Productos Sanitarios. Informe mensual sobre Medicamentos de Uso humano y Productos sanitarios septiembre 2014. Disponible en: http://www.aemps.gob.es/informa/boletinMensual/2014/septiembre/boletin-septiembre.htm\#nuevosMed

10. Committee for Medicinal Products for Human Use (CHMP). Balugrastim. Disponible en: http://www.ema.europa.eu/ema/index.jsp?curl=pages/ medicines/human/medicines/002637/smops/Positive/human_smop_000730. jsp\&mid=WC0b01ac058001d127

11. Volovat C., Gladkov OA., Bondarenko IM., Barash S., Buchner A., Bias P. et al: «Efficacy and safety of balugrastim compared with pegfilgrastim in patients with breast cancer receiving chemotherapy.» Clin Breast Cancer. 2014;14(2):101-108. Disponible en: http://www.ncbi.nlm.nih.gov/pub$\mathrm{med} / 24485296$

12. Tashima K., Crofoot G., Tomaka FL., Kakuda TN., Brochot A., Vanveggel S. et al: «Phase IIIb, open-label single-arm trial of darunavir/cobicistat (DRV/ COBI): Week 48 subgroup analysis of HIV-1-infected treatment-naive adults.» J Int AIDS Soc. 2014;17(4 Suppl 3):19772. Disponible en: http://www.ncbi. nlm.nih.gov/pubmed/25397516

13. Committee for Medicinal Products for Human Use (CHMP). Rezolsta. Disponible en: http://www.ema.europa.eu/ema/index.jsp?curl=pages/medicines/human/medicines/002819/smops/Positive/human_smop_000736. jsp\&mid=WC0b01ac058001d 127

14. Feld J.J.,Kowdley K.V., Coakley E., Sigal S., Nelson D.R., Crawford D. et al. Treatment of HCV with ABT-450/r- Ombitasvir and dasabuvir with ribavirin. N Engl J Med 2014;370:1594-1603. Disponible en: http://www.ncbi.nlm.nih. gov/pubmed/?term=24720703

15. Zeuzem S., Jacobson I.M., Baykal T., Marinho R.T., Poordad F., Bourlière M. et al. Retreatment of HCV with ABT-450/r-Ombitasvir and dasabuvir with ribavirin. N Engl J Med 2014;370:1604-1614 .Disponible en: http://www.ncbi. nlm.nih.gov/pubmed/24720679

16. Committe for Medicinal Products for Human Use (CHMP). Disponible en: http://www.ema.europa.eu/docs/en_GB/document_library/Summary_of_opinion_-_Initial_authorisation/human/003837/WC500177625.pdf

17. Committee for Medicinal Products for Human Use (CHMP) http://www.ema. europa.eu/docs/es_ES/document_library/EPAR_Product_Information/human/002825/WC500179470.pdf

18. Lukina E, Watman N, Arreguin EA, Banikazemi M, Dragosky M, Iastrebner $\mathrm{M}$ et al. A phase 2 study of eliglustat tartrate (Genz-112638), an oral substrate reduction therapy for Gaucher disease type 1. Blood. 2010;116(6):893-899. Disponible en: http://www.ncbi.nlm.nih.gov/pmc/articles/PMC2924227/

19. Lukina E, Watman N, Dragosky M, Pastores GM, Arreguin EA, Rosenbaum $\mathrm{H}$ et al. Eliglustat, an investigational oral therapy for Gaucher disease type 1: Phase 2 trial results after 4 years of treatment.Blood Cells $\mathrm{Mol}$ Dis. 2014 ;53(4):274-276. Disponible en: http://www.ncbi.nlm.nih.gov/pubmed/24835462

20. Committe for Medicinal Products for Human Use (CHMP). Disponible en: http://www.ema.europa.eu/docs/en_GB/document_library/Summary_of_opinion_-_Initial_authorisation/human/003724/WC500177564.pdf 


\section{Evaluación positiva de medicamentos: septiembre/octubre/noviembre 2014}

21. Duavive ${ }^{\mathbb{2}}$, ficha técnica de la Agencia Europea de Medicamentos. Disponible en: http://ec.europa.eu/health/documents/communityregister/2014/20141216130229/anx_130229_es.pdf

22. Lobo RA., Pinkerton JV., Gass ML., Dorin MH., Ronkin S. , Pickar JH. et al: «Evaluation of bazedoxifene/ conjugated estrogens for the treatment of menopausal symptoms and effects on metabolic parameters and overall safety profile.» Fertil Steril. 2009;92(3):1025-1038. Disponible en: http://www.ncbi. nlm.nih.gov/pubmed/19635615

23. Agencia Española de Medicamentos y Productos Sanitarios. Informe mensual sobre Medicamentos de Uso Humano y Productos Sanitarios Septiembre 2014. Disponible en: http://www.aemps.gob.es/informa/boletinMensual/2014/ septiembre/docs/boletin-mensual_septiembre-2014.pdf

24. Committee for Medicinal Products for Human Use (CHMP). Ketoconazol. Disponible en:http://www.ema.europa.eu/ema/index.jsp?curl=pages/news_and_ events/news/2014/09/news_detail_002174.jsp\&mid=WC0b01ac058004d5c1

25. Committee for Medicinal Products for Human Use (CHMP). Moventig. Disponible en: http://www.ema.europa.eu/docs/en_GB/document_library/Summary_of_opinion_-_Initial_authorisation/human/002810/WC500173691.pdf

26. Chey WD., Webster L., Sostek M., Lappalainen .J, Barker PN., Tack J. et al: «Naloxegol for opioid-induced constipation in patients with noncancer pain. «N Engl J Med. 2014; 370(25):2387-2396. Disponible en: http://www.ncbi.nlm. nih.gov/pubmed/24896818

27. VARGATEF .FICHA TÉCNICA. Disponible en: http://www.ema.europa.eu/ docs/es_ES/document_library/EPAR_-_Product_Information/human/002569/ WC500179970.pdf

28. Committee for Merdicinal Products for Human Use (CHMP). Disponible en: http://www.ema.europa.eu/docs/en_GB/document_library/Summary_of_opinion_-_Initial_authorisation/human/002569/WC500173607.pdf

29. Richeldi L, du Bois RM, Raghu G, Azuma A, Brown KK, Costabel U et al. INPULSIS Trial Investigators. Efficacy and safety of nintedanib in idiopathic pulmonary fibrosis. N Engl J Med. 2014;370(22):2071-2082. Disponible en : http://www.nejm.org/doi/full/10.1056/NEJMoa1402584

30. Committe for Medicinal Products for Human Use (CHMP). Disponible en: http://www.ema.europa.eu/docs/en_GB/document_library/Summary_of_opinion_-_Initial_authorisation/human/003821/WC500177632.pdf

31. Windyga J., Lissitchkov T., Stasyshyn O., Mamonov V., Rusen L., Lamas JL. et al: «Pharmacokinetics, efficacy and safety of BAX326, a novel recombinant factor IX: a prospective, controlled, multicentre phase I/III study in previously treated patients with severe (FIX level $<1 \%$ ) or moderately severe (FIX level $\leq 2 \%$ ) haemophilia B.» Haemophilia. 2014; 20(1):15-24. Disponible en: http:// www.ncbi.nlm.nih.gov/pubmed/?term=NONACOG+GAMMA

32. Rixubis, ficha técnica de la Administración de Alimentos y Medicamentos de EE.UU Disponible en: http://www.baxter.com/downloads/healthcare_professionals/products/RIXUBIS_PI.pdf

33. Ledermann J., Harter P., Gourley C., Friedlander M., Vergote I., Rustin G. et al: «Olaparib maintenance therapy in patients with platinum-sensitive relapsed serous ovarian cancer: a preplanned retrospective analysis of outcomes by BRCA status in a randomised phase 2 trial.» Lancet Oncol. 2014;15(8):852861. Disponible en: http://www.ncbi.nlm.nih.gov/pubmed/24882434

34. Lynparza. FDA Briefing Document. Oncologic Drugs Advisory Committee Meeting, June 25, 2014. Disponible en: http://www.fda.gov/downloads/AdvisoryCommittees/CommitteesMeetingMaterials/Drugs/OncologicDrugsAdvisoryCommittee/UCM402207.pdf

35. Resumen opinión del EMA/CHMP/688255/2014 de 20 de noviembre de 2014. Disponible en: http: http://www.ema.europa.eu/docs/en_GB/document_library/Summary_of_opinion_-_Initial_authorisation/human/003839/ WC500177626.pdf

36. Wurz GT, Kao CJ, DeGregorio MW. Safety and efficacy of ospemifene for the treatment of dyspareunia associated with vulvar and vaginal atrophy due to menopause. Clin Interv Aging. 2014 Nov 13;9:1939-50. doi: 10.2147/ CIA.S73753. eCollection 2014.Disponible en: http://www.ncbi.nlm.nih.gov/ pubmed/25419123

37. Committe for Medicinal Products for Human Use (CHMP). Disponible en: http://www.ema.europa.eu/docs/en_GB/document_library/Summary_of_opinion_-_Initial_authorisation/human/002780/WC500177633.pdf

38. Fuchs CS., Tomasek J., Yong CJ., Dumitru F., Passalacqua R., Goswami C. et al: «Ramucirumab monotherapy for previously treated advanced gastric or gastro-oesophageal junction adenocarcinoma (REGARD): an international, randomised, multicentre, placebo-controlled, phase 3 trial.» Lancet. 2014; 383(9911):31-39. Disponible en: http://www.ncbi.nlm.nih.gov/pubmed/?term= RAMUCIRUMAB+gastric

39. Langley RG, Elewski BE, Lebwohl M, Reich K, Griffiths CE, Papp K et al ERASURE Study Group; FIXTURE Study Group. Secukinumab in plaque psoriasis--results of two phase 3 trials. N Engl J Med. 2014;371(4):326-338. Disponible en: http://www.nejm.org/doi/full/10.1056/NEJMoa1314258

40. Ohtsuki M, Morita A, Abe M, Takahashi H, Seko N, Karpov A et al. Secukinumab efficacy and safety in Japanese patients with moderate-to-severe plaque psoriasis: Subanalysis from ERASURE, a randomized, placebo-controlled, phase 3 study. J Dermatol 41(12):1039-1046. December 2014 Disponible en: http://onlinelibrary.wiley.com/doi/10.1111/1346-8138.12668/full

41. Committe for Medicinal Products for Human Use (CHMP). Disponible en: http://www.ema.europa.eu/docs/en_GB/document_library/Summary_of_ opinion_-_Ini tial_authorisation/human/003729/WC500177620.pdf

42. Harvoni®, Ficha Técnica de la Agencia Europea de Medicamentos. Disponible en: http://www.ema.europa.eu/docs/es_ES/document_library/ EPAR_-_Product_Information/human/003850/WC500177995.pdf

43. Afdhal N., Zeuzem S., Kwo .P, Chojkier M., Gitlin N., Puoti M. et al: «Ledipasvir and sofosbuvir for untreated HCV genotype 1 infection.» N Engl J Med. 2014; 370(20):1889-1898 Disponible en: http://www.ncbi.nlm.nih.gov/ pubmed/24725239

44. Afdhal N., Zeuzem S., Kwo .P, Chojkier M., Gitlin N., Puoti M. et al: «Ledipasvir and sofosbuvir for previously treated $\mathrm{HCV}$ genotype 1 infection.» $\mathrm{N} \mathrm{Engl}$ J Med. 2014; 370(16):1483-1493. Disponible en: http://www.ncbi.nlm.nih.gov/ pubmed/24725238

45. Lymphoseek, Ficha Técnica de la Agencia Europea de Medicamentos. Disponible en: http://ec.europa.eu/health/documents/communityregister/2014/20141119129982/anx_129982_es.pdf

46. Wallace AM., Han LK., Povoski SP., Deck K., Schneebaum S., Hall NC. et al: «Comparative evaluation of $[(99 \mathrm{~m})$ tc]tilmanocept for sentinel lymph node mapping in breast cancer patients: results of two phase 3 trials.» Ann Surg Oncol. 2013;20(8):2590-2599. Disponible en: http://www.ncbi.nlm.nih.gov/ pubmed/23504141

47. Marcinow AM., Hall N. , Byrum E., Teknos TN., Old MO., Agrawal A. et al: «Use of a novel receptor-targeted (CD206) radiotracer, 99mTc-tilmanocept, and SPECT/CT for sentinel lymph node detection in oral cavity squamous cell carcinoma: initial institutional report in an ongoing phase 3 study.» JAMA Otolaryngol Head Neck Surg. 2013;139(9):895-902. Disponible en: http://www. ncbi.nlm.nih.gov/pubmed/24051744

48. Resumen opinión del EMA/CHMP/666694/2014 de 20 de noviembre de 2014. Disponible en: http://www.ema.europa.eu/docs/en_GB/document_library/ Summary_of_opinion_-_Initial_authorisation/human/002814/WC500177579. pdf

49. Tricoci P, Huang Z, Held C, Moliterno D, Armstrong P, Van de Werf F, et al. Thrombin-receptor antagonist Vorapaxar in acute coronary syndromes. N Engl J Med 2012; 366: 20-33.

50. Morrow D, Braunwald E, Bonaca M, Ameriso S, Dalby A, Polly M, et al. Vorapaxar in the secondary prevention of atherothrombotic events. N Engl J Med 2012; 366:1404-1413. 